\title{
The Brazillian version of the hand mobility in scleroderma (HAMIS) test: translation and validation
}

\author{
Pedro Ming Azevedo ${ }^{1 *}$ D, Estefânia Sartorato Sanson ${ }^{1}$, Thelma Larocca Skare', \\ Thiago Alberto Fernandes Gomes dos Santos ${ }^{1}$ and Patricia Martin ${ }^{1,2}$
}

\begin{abstract}
Background: The Hand Mobility in Scleroderma (HAMIS) test was created to measure the degree of dysfunction of hand movements imposed by systemic sclerosis (SSc). The modified version (mHAMIS), with 4 of the 9 original items, was developed later. The goal of the present study was to translate and validate HAMIS and mHAMIS into Brazilian Portuguese and culture.

Methods: After direct and reverse translation and comprehension test in 10 SSC patients, HAMIS-Br was applied to another 32 patients with SSc. To evaluate internal consistency, intraobserver and interobserver agreement, and intraobserver and interobserver reliability, we used respectively the Cronbach's a coefficient, kappa concordance and intraclass correlation (ICC). The correlation between HAMIS-Br and mHAMIS-Br was evaluated and a factorial analysis was performed.

Results: HAMIS-Br showed excellent internal consistency (Cronbach's a = 0.997), good intraobserver agreement (kappa between 0.78 [95\% Cl $=0.57-0.99]$ and 1$)$ and intraobserver and interobserver reliability $($ ICC $=0.993,95 \% \mathrm{Cl}=$ $0.973-0.993$ and ICC $=0.994,95 \% \mathrm{Cl}=0.987-0.997$, respectively). The mHAMIS-Br presented similar results and excellent correlation with HAMIS-Br $(r=0.923)$. The factorial analysis extracted three groups of questions that explain $84.4 \%$ of the total variance, and that can be understood through the influence of certain movements in the interpretation of others: [1] questions whose interpretation is influenced by the extension of the fingers, [2] questions whose interpretation is influenced by flexion of the fingers, [3] volar flexion of the fingers, with similar correlation with both other factors.

Conclusions: HAMIS-Br and mHAMIS-Br showed good agreement, intraobserver and interobserver reliability, and internal validity. It is necessary to be attentive to the influence of certain limitations of movements in the interpretation of others.
\end{abstract}

Keywords: Systemic sclerosis, HAMIS, Dysfunction, Hands, Portuguese, Brazil

\section{Background}

Systemic sclerosis (SSc) is a connective tissue disease characterized by autoimmunity, vasculopathy and fibrosis of skin and internal organs [1]. Fibrosis leads to thickening of the skin, causing decreased mobility in various segments of the body [1]. This process begins and is more intense in the distal limb segments, which

\footnotetext{
* Correspondence: pedroming@gmail.com

${ }^{1}$ Department of Rheumatology at Mackenzie Evangelical School of Medicine,

R. Padre Anchieta 2770, Curitiba, Paraná 80730-000, Brazil

Full list of author information is available at the end of the article
}

is why the hand is especially affected. About $90 \%$ of patients with SSc refer to difficulty in holding objects $[2,3]$. Hands are of particular importance for work, intellectual, artistic and leisure activities of the human being. Thus, conditions that affect the functioning of this segment of the body tend to have a huge impact on patients' quality of life [3].

In order to measure the degree of dysfunction of hand movements imposed by SSc, it is necessary to have specific instruments. HAMIS (Hand Mobility in Scleroderma) serves this purpose and has often been used in

C The Author(s). 2019 Open Access This article is distributed under the terms of the Creative Commons Attribution 4.0 International License (http://creativecommons.org/licenses/by/4.0/), which permits unrestricted use, distribution, and reproduction in any medium, provided you give appropriate credit to the original author(s) and the source, provide a link to the Creative Commons license, and indicate if changes were made. The Creative Commons Public Domain Dedication waiver (http://creativecommons.org/publicdomain/zero/1.0/) applies to the data made available in this article, unless otherwise stated. 
that sense in the countries in which it was translated. The instrument proposed by two Swedish authors evaluates specific movements of the hands necessary for the activities of daily living and for the instrumental activities of quotidian [2].

In previous studies HAMIS proved to be able to adequately discriminate the hand function of SSc patients compared to those without this condition with high interobserver and intraobserver reliability $[4,5]$. The test was also significantly correlated with thickening of the skin of the hands, both in cross-sectional and longitudinal follow-up studies, with the impact of the disease on the daily activities of these patients $[2,5-7]$ and with organ involvement in SSc [8].

In 2014, in order to simplify the evaluation tool, the HAMIS authors developed mHAMIS (modified HAMIS), a smaller version of the test, composed of 4 of the 9 original items. Modified HAMIS demonstrated a good correlation with the original HAMIS, the modified Rodnan skin score (MRSS) and the hand cutaneous score, as well as the ability to discriminate between limited and diffuse SSc in the first 3 years of the disease [9]. In the early stages of SSc, mHAMIS reflects the activity of the cutaneous disease, and in later stages it can be considered as a measure of the damage resulting from fibrotic and vascular involvement [9].

Despite proving its value in clinical follow-up and research involving SSc patients, neither HAMIS nor its minor version, mHAMIS, had been translated or validated into the Brazilian language or culture. To this end, the present work was conceived.

\section{Methods}

After approval by the ethics committee of the local institution and signing of the informed consent, all patients from the SSc outpatient clinic with the systemic forms of the disease were invited to participate in the study. Patients with non-SSc related morbidities that could eventually cause hand dysfunction, such as sequelae of previous trauma, neurological disease, or clinically significant osteoarthritis were excluded. The process took place in three phases: translation into Portuguese, intercultural adaptation and interobserver and intraobserver validation.

\section{Instruments: HAMIS and mHAMIS}

HAMIS evaluates 9 specific hand movements including finger flexion, finger extension, thumb abduction, pincer grip, finger abduction, volar flexion, dorsal extension, pronation and supination. To do this it uses objects with standardized measures such as pencils, cutlery handle, coffee packs, milk cartons, line spool, and a table. Each movement is graded on a scale of zero to three, with zero reflecting the normal movement and three the total inability to perform the movement. The final score is given by the sum of the graduation obtained in each movement, the worst score being 27 [4].

Modified HAMIS evaluates 4 of the 9 movements evaluated by HAMIS (finger flexion, finger extension, abduction of the fingers and dorsal extension of the hands), using a similarly graded scale, the worst score being 12 [9].

\section{Translation into Brazilian Portuguese}

At first, two of the authors of the present study, Brazilian, experienced rheumatologists and fluent in English, translated the original instrument from English into Brazilian Portuguese. This translation was then sent to two English language natives' residing in Brazil and fluent in Brazilian Portuguese for reverse translation (from Portuguese to English). The product of the reverse translation was then compared with the initial questionnaire in search of meaning mismatches. Any discrepancies were corrected by consensus among the four translators [5].

\section{Intercultural adaptation}

The final instrument obtained was applied to a group of 10 patients from a SSc outpatient clinic, in search of incongruities or difficulties of adaptation / comprehension in that population. Items considered problematic for $20 \%$ or more of these patients were rewritten by the researchers and the new version was reapplied to a second group of 10 patients, which allowed the final version of the instrument to be written, henceforth called HAMIS$\mathrm{Br}$ (Additional file 1).

\section{Interobserver and intraobserver validation}

Two researchers, a previously trained medical student and an experienced rheumatologist, applied HAMIS$\mathrm{Br}$ to one hand of patients of a third group, consisting of 32 consecutives clinically stable SSc patients. To verify intraobserver reproducibility, or test-retest, the first investigator applied the instrument on day 1 and repeated it on day 15. During this interval there was no change in patients' treatment. The second examiner applied the questionnaire to the same patients only on day 15 , in order to verify interobserver reproducibility. The internal consistency of HAMIS-Br and mHAMIS-Br and correlation between the two instruments were also evaluated.

\section{Statistical analysis}

Data were analyzed using the IBM SPSS Statistics v.20.0 software. Armonk, NY: IBM Corp. The results of quantitative variables were described by means, medians and 
amplitudes. For categorical variables, frequencies and percentages were presented.

Associations were tested between the HAMIS-Br and mHAMIS, as well as between the HAMIS-Br and mHAMIS-Br scores and the clinical parameters that could possibly modify the results of these tests: duration of disease to be counted from first non-Raynaud symptom, disease duration from onset of Raynaud, digital ulcers, pitting scars, type of disease (diffuse versus limited), arthritis, calcinosis, hand asymmetry and MRSS.

To evaluate the association between two quantitative variables, Spearman correlation coefficients were estimated, and their significance assessed. An excellent correlation was considered if $r>0.91$; good ratio if $r$ between 0.90 and 0.71 ; moderate ratio if $\mathrm{r}$ between 0.51 and 0.7; poor ratio if $r$ between 0.50 and 0.31 and absent if $\mathrm{r}<0.30$.

The comparison between 2 groups was done using the non-parametric Mann-Whitney test. The option for non-parametric tests is justified by the nature of the evaluated variables (scores). Values of $p<0.05$ indicated statistical significance.

The intraobserver and interobserver agreement of each movement evaluated by the instrument was verified by means of the kappa concordance coefficient and its 95\% confidence interval (95\% CI). If kappa was between 0.81 and 1 the agreement was considered great; if between 0.61 and 0.80 it was considered good; if between 0.41 and 0.6 it was considered moderate; if between 0.21 and 0.40 it was considered regular and if kappa was between 0 and 0.20 it was considered low [2].

The concordance (or reliability) of the continuous variables (final score given by the sum of the scores obtained for each movement) for HAMIS-Br and mHAMIS-Br was evaluated by the intraclass correlation coefficient (ICC) and its IC 95\%. For this purpose, the bidirectional mixed model with a single classification was used, taking into account the consistency. We considered ICC $>0.90$ of excellent reliability; ICC between 0.75 and 0.9 of good reliability; ICC between 0.5 and 0.75 of moderate and ICC $<0.50$ of low reliability $[4,10]$.

To evaluate the internal consistency, the Cronbach's alpha coefficient was used. An instrument is considered to have very good internal consistency if Cronbach's alpha greater than 0.9 ; if between 0.8 and 0.9 the internal consistency will be good; if between 0.7 and 0.8 will be reasonable; if between 0.6 and 0.7 will be weak and if less than 0.6 will be inadmissible [11].

Factor analysis was used to evaluate the factorial structure of the instrument. The analysis model considered principal components with factors corresponding to eigenvalues between 0.8 and 5.6 and standardized varimax rotation [12].

\section{Results}

The instrument was applied to 32 SSc patients aged 24 to 74 years (median 55 years), most of whom had late disease (median disease time from first non-RP symptom of 10.25 years). The median of MRSS was 3, ranging from 0 to 29 , but the score was not calculated in 6 patients. Additional clinical characteristics of patients are shown in Table 1. Calcinosis and hand asymmetry were not present in enough numbers to allow association tests and in one patient the presence of arthritis was not evaluated.

The Brazilian version of HAMIS (Additional file 1) showed good intraobserver and interobserver agreement in all items of the questionnaire, as shown in Table 2. Although the examiners disagreed on the results of item 8 in only two patients, the statistical method was not able to detect interobserver agreement since the answers were limited to categories 1 and 2 .

Regarding the final score, given by the sum of all items of the instrument, intraobserver and interobserver reliability was also excellent for both HAMIS-Br and mHAMIS-Br, as displayed in Table 3.

Both instruments showed excellent internal consistency (Cronbach's alpha coefficient of 0.994 for HAMIS-Br and 0.997 for mHAMIS-Br). The correlation between the two instruments was excellent $(r=0.923$, $p<0.0001$ ).

No correlation was found between the MRSS score with the final HAMIS- $\mathrm{Br}(\mathrm{r}=0.255, p=0.209)$ or with mHAMIS-Br scores $(r=0.325, p=0.106)$. The fact that MRSS could not be tested in 6 patients may have influenced this outcome. Also, no association was found between the two HAMIS-Br versions and clinical parameters such as form of disease, arthritis, pitting

Table 1 Clinical characteristics of patients

\begin{tabular}{lll}
\hline Variable & $\mathrm{N}$ & $\%$ \\
\hline Female gender & 25 & 78.12 \\
Limited type of the disease & 16 & 50 \\
Asymmetry of hands & 1 & 3.12 \\
Digital Ulcers & 3 & 9.74 \\
Pitting scars & 11 & 34.37 \\
Calcinosis in hands $_{\text {Arthritis }}{ }^{a}$ & 2 & 6.25 \\
Pulmonary fibrosis & 12 & 38.70 \\
Esophageal dysmotility & 17 & 53.12 \\
Pulmonary arterial hypertension & 19 & 59.37 \\
\hline
\end{tabular}

ain one patient, the presence of arthritis was not evaluated. All the other clinical parameters were evaluated in all patients 
Table 2 Intraobserver and interobserver agreement for each evaluated movement

\begin{tabular}{|c|c|c|}
\hline Movement & $\begin{array}{l}\text { Intraobserver agreement } \\
\text { (kappa) }\end{array}$ & $\begin{array}{l}\text { Interobserver agreement } \\
\text { (kappa) }\end{array}$ \\
\hline Finger flexion & 0.90 (Cl 95\% 0.77-1) & 0.80 (Cl 95\% 0.63-0.97) \\
\hline Finger extension & 1 & 1 \\
\hline $\begin{array}{l}\text { Abduction of the } \\
\text { thumb }\end{array}$ & 1 & 0.86 (Cl 95\% 0.71-1) \\
\hline Pincer grip & 0.93 (Cl 95\% 0.81-1) & $0.77(\mathrm{Cl} 95 \% 0.57-0.97)$ \\
\hline $\begin{array}{l}\text { Abduction of the } \\
\text { fingers }\end{array}$ & 0.92 (Cl 95\% 0.77-1) & 1 \\
\hline Volar flexion & 0.78 (Cl 95\% 0.57-0.99) & $0.81(\mathrm{Cl} 95 \% 0.61-1)$ \\
\hline Dorsal extension & 0.90 (Cl 95\% 0.73-1) & $0.91(\mathrm{Cl} 95 \% 0.73-1)$ \\
\hline Pronation & 1 & - \\
\hline Supination & 1 & $0.93(\mathrm{Cl} 95 \%$ 0.78-1) \\
\hline
\end{tabular}

${ }^{*} p<0,0001$ for all movements evaluated; $\mathrm{Cl}$ Confidence interval

scars, digital ulcers (Table 4) or duration of the disease (Table 5).

The factorial analysis extracted three factors that could be characterized clinically and that explain $84.4 \%$ of the total variance. The first factor added finger extension, finger abduction, dorsal extension, and supination movements, whose assessments can be influenced by the incapacity of extending completely the fingers. The second factor added the movements of flexion of the fingers, abduction of the thumb and pincer grip, whose evaluations can be influenced by difficulties in the flexion of fingers. The volar flexion of fingers showed a similar correlation with factor 1 (extension) and factor 2 (flexion). Pronation stood isolated as a distinct factor. The first 2 factors showed excellent internal consistency (Cronbach's alpha = 0.979 to 0.993 ) and intraobserver and interobserver reliability, as displayed in Table 6 .

\section{Discussion}

The cross-cultural translation and validation of HAMIS for Portuguese spoken in Brazil was carried out in the present study, as well as the evaluation of the reproducibility and internal consistency of the new versions. In addition, factorial analysis of HAMIS-Br was performed with the isolation of 3 factors.

The present version of HAMIS (HAMIS-Br) is the first adaptation to the Brazilian language and culture of this

Table 3 Intraobserve and interobserver reliability for HAMIS-Br and $\mathrm{mHAMIS-Br}$

\begin{tabular}{lll}
\hline Instrument & ICC Intraobserver & ICC interobserver \\
\hline HAMIS & $0.993($ IC95\% 0.973-0.993)* & 0.994 (IC95\% 0.987-0.997)* \\
HAMISm & $0.997($ IC95\% 0.994-0.999)* & $0.997($ IC95\% 0.993-0.998)* \\
\hline
\end{tabular}

${ }^{*} p<0.0001 ; C l$ Confidence interval instrument for objective evaluation of the dysfunction of the hands of SSc patients, and our results point to a high reliability of the method. Each of the HAMIS-Br items proved to be excellent in the intraobserver and interobserver agreement, except for the pronation movement (item 8 of the instrument), in which the statistical method was unable to assess interobserver agreement because all patients were able to perform the movement satisfactorily and the answers were limited to categories 1 and 2. It should be highlighted that this last finding does not weaken our results. The same phenomenon was observed by Sandqvist and Eklund when evaluating the intraobserver and interobserver agreement of the original HAMIS. Unlike these authors, who observed moderate agreement for supination, we found excellent agreement for this movement [2].

Since mHAMIS is derived from 4 of the 9 original HAMIS questions, the Brazilian-Portuguese version of mHAMIS (mHAMIS-Br) can be extrapolate from the HAMIS-Br. The mHAMIS is composed of items related to flexion of the fingers, extension of the fingers, abduction of the fingers and the dorsal extension of the hands. All these movements had excellent interobserver and interobserver agreement in our work.

With respect to the final score of HAMIS-Br and mHAMIS-Br, the sum of the scores obtained in each individually assessed movement, we also saw excellent internal consistency and intraobserver and interobserver reliability, similar to what was observed in the validation of the Italian version of the instrument [5] and better than what was described by Sandqvist [4].

Of the numerous dysfunctions caused by SSc, those related to cutaneous fibrosis of the hands are among the most precocious and limiting. Any study that intends to evaluate the impact of the disease in these patients needs objective means to measure the limitation of the movements that the disease imposes in their hands. While MRSS is almost universally accepted as a measure of cutaneous edema, which is believed to be related to the disease activity in the skin [13], HAMIS has been emerging internationally as an instrument to measure the impact of the disease on hand function, largely related to damages from fibrotic and vascular involvement [9]. In our study, it was observed that the median MRSS score was low and did not correlated to the degree of hand involvement evaluated by HAMIS-Br or mHAMIS-Br. This may have happened because all our patients had late-onset disease. The finding reinforces the importance of HAMIS as an instrument to evaluate hand function, regardless of the values of the MRSS. We observed no difference between the values of HAMIS$\mathrm{Br}$ and mHAMIS-Br among patients with digital ulcers, which may have occurred due to the low 
Table 4 Clinical parameters association test with HAMIS-Br and mHAMIS-Br

\begin{tabular}{|c|c|c|c|c|c|c|c|c|c|}
\hline \multirow[t]{2}{*}{ Clinical parameters } & & \multicolumn{4}{|c|}{ HAMIS-Br } & \multicolumn{4}{|c|}{ mHAMIS-Br } \\
\hline & & Mean & Median & Range & $p$ & Mean & Median & Range & $p$ \\
\hline \multirow[t]{2}{*}{ Form of disease } & Diffuse SSC & 6.13 & 3 & 0-19 & 0.401 & 3.07 & 1.0 & $0-10$ & 0.470 \\
\hline & Limited SSC & 3.00 & 2 & $0-15$ & & 1.88 & 1.0 & $0-8$ & \\
\hline \multirow[t]{2}{*}{ Digital ulcers } & Presence & 11 & 14 & 0-19 & 0.315 & 6 & 8 & $0-10$ & 0.251 \\
\hline & Absence & 4.12 & 2 & $0-15$ & & 1.92 & 1 & $0-9$ & \\
\hline \multirow[t]{2}{*}{ Pitting Scars } & Presence & 6.42 & 4 & 0-19 & 0.435 & 3.08 & 1.5 & $0-10$ & 0.704 \\
\hline & Absence & 4.11 & 2 & $0-15$ & & 2.05 & 1.0 & $0-9$ & \\
\hline \multirow[t]{2}{*}{ Arthritis } & Presence & 4.79 & 2 & 0-19 & 0.704 & 2.92 & 1.5 & $0-9$ & 0.346 \\
\hline & Absence & 5.33 & 4 & $0-15$ & & 2.16 & 1 & $0-10$ & \\
\hline
\end{tabular}

number of patients with active ulcers in our study. In addition, patients with pitting scars did not present higher scores for HAMIS-Br or mHAMIS-Br, possibly because scars are not always painful, therefore may not impact hand function. Of note, the presence of arthritis also had no impact on HAMIS or mHAMIS results.

Factorial analysis is a statistical method that seeks patterns in the responses, which allow the grouping of the items into blocks, called 'factors'. In this context, it is interesting to note that all movements tested by items associated with the first factor (finger extension, finger abduction, dorsal extension and supination) may have their interpretation affected by difficulties in the complete extension of the fingers. In the same way, the interpretation of the movements tested by items in the second factor (flexion of the fingers, abduction of the thumb and pincer grip) can be significantly affected by deficiencies in the flexion of the fingers. For example, supination capacity may wrongly appear to be diminished if the fingers do not touch the table because of restrictions on the extension of fingers. Likewise, the patient may not be able to pick up a milk carton because he/she cannot flex his fingers and not because he/she cannot lift his thumb. The nature of the problem seems to be the subjective evaluation of each movement. We believe that the creators of the HAMIS test deliberatively chose it to be this way to make the test faster, easier and more intuitive, which, in a way, was achieved, but not without counterparts. The possibility of one movement deficiency influencing another is the main one and is being highlighted by the present work. The accuracy of HAMIS is impaired if the examiners consider the answers in a literal and naive way. Rather, they should pay attention to the movement being tested and seek to disregard failures in reaching the goal when these failures are secondary to limitations of other movements. It is important to add that this caveat is valid for any version of HAMIS, and not exclusive to the Brazilian version.

Among the limitations of our study are the absence of external validation of HAMIS-Br, that is, the comparison with other instruments that evaluate hand function. However, the original version proposed by Sandqvist showed concurrent validity of HAMIS in comparison to range of motion and cutaneous score for all movements, except for forearm pronation and supination [4]. At the same time, the Italian version of the instrument showed good correlation with the Cochin Hand Function Scale (CHFS) and the ipsilateral closure and opening of the hand [5]. Another limitation was the evaluation of the movements in only one hand, since the application of the test in both hands would help the interobserver and intraobserver validation. At the same time, the present study followed the methodology of Sandqvist et al., who preferred to use only the dominant hand in the analysis of the data collected in the original HAMIS validation study [2].

Table 5 Correlation between disease duration and $\mathrm{HAMIS-Br}$ and $\mathrm{mHAMIS-Br}$

\begin{tabular}{|c|c|c|c|c|c|c|c|}
\hline & \multicolumn{3}{|c|}{ Time (years) } & \multicolumn{2}{|l|}{ HAMIS-Br } & \multicolumn{2}{|l|}{ mHAMIS-Br } \\
\hline & mean & median & range & $r$ & $p$ & $r$ & $p$ \\
\hline Disease time from RP & 11.34 & 10 & $4-24$ & $r=-0.201$ & 0.278 & $r=-0.163$ & 0.381 \\
\hline Disease time from 1st NRPS & 10.25 & 9 & $0-24$ & $r=-0.175$ & 0.345 & $r=-0.151$ & 0.418 \\
\hline
\end{tabular}

RP Raynaud phenomenon, NRPS Non- Raynaud phenomenon symptom 
Table 6 Intraobserver and interobserver reliability for HAMIS-Br factors 1 and 2

\begin{tabular}{lll}
\hline Factor & ICC Intraobserver & ICC interobserver \\
\hline 1 - Extension & $0.998($ IC95\% 0.997-0.999)* & $0.996($ IC95\% 0.991-0.998)* \\
2- Flexion & $0.993($ IC95\% 0.986-0.997)* & $0.979($ ICC95\% 0.957-0.990)* \\
\hline${ }^{*} p<$ 0.0001; ICC Intraclass correlation coefficient &
\end{tabular}

\section{Conclusions}

In conclusion, HAMIS-Br and mHAMIS-Br showed good reliability and internal validity and can be used for studies evaluating hand movements in patients with SSc, provided that care is taken to assess exclusively the movement evaluated by each item, in order to avoid misinterpretations of movements.

\section{Supplementary information}

Supplementary information accompanies this paper at https://doi.org/10. 1186/s42358-019-0093-5.

Additional file 1. The Brazillian version of the hand mobility in scleroderma (HAMIS-Br).

\section{Abbreviations}

CHFS: Cochin Hand Function Scale; Cl: Confidence interval; HAMIS: Hand mobility in Scleroderma; ICC: Intraclass correlation coefficient; mHAMIS: Modified Hand Mobility in Scleroderma; MRSS: modified Rodnan skin score; NRPS: Non-Raynaud phenomena symptom; RP: Raynaud phenomena; SCc: Systemic Sclerosis

\section{Acknowledgements}

Our sincere thanks to Márcia Olandoski, who kindly assisted us in the statistical analysis and to Professor Gunnel Sandqvist who kindly ceded the rights to use the original HAMIS and MHAMIS photos.

\section{Authors' contributions}

PMA, PM and TLS idealized wrote and approved the project. The same authors were responsible for the translation of the original HAMIS. PMA and ESS applied the HAMIS-Br to patients. PM performed the statistical analysis and directly participated in writing the manuscript, together with TAFGS, and PMA. All authors read and approved the final manuscript.

\section{Funding}

The present work was financed by the authors themselves.

Ethics approval and consent to participate.

The present study was approved by the Ethics Committee of the Sociedade Evangélica Beneficente de Curitiba at 17th May 2009, under the reference number 3246/09.

\section{Availability of data and materials}

The datasets used and/or analysed during the current study are available from the corresponding author on reasonable request.

\section{Consent for publication}

Not applicable.

\section{Competing interests}

The authors declare that they have no competing interests.

\section{Author details}

'Department of Rheumatology at Mackenzie Evangelical School of Medicine, R. Padre Anchieta 2770, Curitiba, Paraná 80730-000, Brazil. ' ${ }^{2}$ Medical School of the Pontifical Catholic University of Paraná. R. Imaculada Conceição, 1155 -

Prado Velho, Curitiba, PR 80215-901, Brazil.
Received: 5 June 2019 Accepted: 30 October 2019

Published online: 21 November 2019

\section{References}

1. Desbois AC, Cacoub P. Systemic SScerosis: An update in 2016. Autoimmun Rev. 2016;15(5):417-26.

2. Sandqvist G, Eklund M. Validity of HAMIS: a test of hand mobility in Scleroderma. Arthritis Care Res. 2000;13(6):382-7 Cited 2019 May 20; Available from: http://www.ncbi.nlm.nih.gov/pubmed/14635314.

3. Sandqvist G, Eklund M, Åkesson A, Nordenskiöld U. Daily activities and hand function in women with scleroderma. Scand J Rheumatol. 2004; 33(2):102-7

4. Sandqvist G, Eklund M. Hand Mobility in Scleroderma (HAMIS) test: the reliability of a novel hand function test. Arthritis Care Res. 2000;13(6):369-74 Cited 2019 May 20; Available from: http://www.ncbi.nlm.nih.gov/ pubmed/14635312.

5. Del Rosso A, Bongi SM, Sigismondi F, Miniati I, Bandinelli F, Matucci-Cerinic M. The Italian version of the Hand Mobility in Scleroderma (HAMIS) test: Evidence for its validity and reliability. Clin Exp Rheumatol. 2010;28(5 SUPPL 62):S42-7.

6. Hesselstrand R, Nilsson JA, Sandqvist G. Psychometric properties of the Swedish version of the scleroderma health assessment questionnaire and the Cochin hand function scale in patients with systemic sclerosis. Scand J Rheumatol. 2013;42(4):317-24.

7. Sandqvist G, Hesselstrand R, Eberhardt K. A longitudinal follow-up of hand involvement and activities of daily living in early systemic sclerosis. Scand J Rheumatol. 2009;38(4):304-10.

8. Ingegnoli F, Boracchi P, Ambrogi F, Gualtierotti R, Galbiati V, Meroni PL. Hand impairment in systemic sclerosis: association of different hand indices with organ involvement. Scand J Rheumatol. 2010;39(5):393-7.

9. Sandqvist G, Wuttge DM, Hesselstrand R. The modified hand mobility in scleroderma test and skin involvement - a followup study. J Rheumatol. 2016;43(7):1356-62.

10. Koo TK, Li MY. A guideline of selecting and reporting Intraclass correlation coefficients for reliability research. J Chiropr Med. 2016;15(2):155-63.

11. Pestana MH, Gageiro JN. Análise de Dados para Ciências Sociais. A Complementaridade do SPSSC. $6^{\text {a }}$ Edição Edição revista e aumentada. $5^{a}$ Edição Revista E Corrigida. 2008. 527-528.

12. Johnson RA, Richard A, Wichern DW. Applied multivariate statistical analysis. 6th ed. New Jersey: Pearson Prentice Hall; 2007. p. 481-538.

13. Khanna D, Furst DE, Clements PJ, Allanore Y, Baron M, Czirjak L, et al. Standardization of the M-Rodnan Skin Score for Use in Clinical Trials of Systemic Sclerosis. J Scleroderma Relat Disord. 2017;2(1):11-8.

\section{Publisher's Note}

Springer Nature remains neutral with regard to jurisdictional claims in published maps and institutional affiliations. 